\title{
Plane strain deformation of a multi-layered poroelastic half-space by surface loads
}

\author{
SARVA Jit Singh ${ }^{1, *}$ and Sunita RAni ${ }^{2}$ \\ ${ }^{1}$ Senior Scientist, INSA, Department of Mathematics, University of Delhi, South Campus, \\ New Delhi 110 021, India. \\ *e-mail: s_j_singh@yahoo.com \\ ${ }^{2}$ Department of Mathematics, Guru Jambheshwar University, Hisar 125 001, India. \\ e-mail: s_b_rani@rediffmail.com
}

The Biot linearized quasi-static theory of fluid-infiltrated porous materials is used to formulate the problem of the two-dimensional plane strain deformation of a multi-layered poroelastic half-space by surface loads. The Fourier-Laplace transforms of the stresses, displacements, pore pressure and fluid flux in each homogeneous layer of the multi-layered half-space are expressed in terms of six arbitrary constants. Generalized Thomson-Haskell matrix method is used to obtain the deformation field. Simplified explicit expressions for the elements of the $6 \times 6$ propagator matrix for the poroelastic medium are obtained. As an example of the possible applications of the analytical formulation developed, formal solution is given for normal strip loading, normal line loading and shear line loading.

\section{Introduction}

Poroelasticity is concerned with heterogeneous media consisting of an elastic solid skeleton infiltrated by a diffusing pore fluid. The theory of poroelasticity studies the time-dependent coupling between the deformation of rock and fluid flow within the rock. The study of quasi-static deformation of a fluid-infiltrated porous half-space by surface loads is important for its geophysical and engineering applications. Biot $(1941,1956)$ developed linearized constitutive and field equations for porous media which have been used very extensively (see, e.g., Rice and Cleary 1976; Bell and Nur 1978; Roeloffs 1988; Kalpna and Chander 1997; Pan 1999). Further references can be found in Wang (2000) and Rudnicki (2001).

Singh (1970) used a generalization of the Thomson-Haskell matrix method to study the deformation of a multi-layered elastic half-space by buried sources. The corresponding two-dimensional problem has been discussed by Singh and Garg
(1985). The formulation of Singh (1970) and Singh and Garg (1985) has been used very extensively. Pan (1999) discussed the deformation of a multilayered poroelastic half-space by buried sources. However, the elements of the propagator matrix given in this paper are complicated functions of the poroelastic parameters used. Wang and Fang (2003) studied the consolidation problem for a multi-layered poroelastic half-space. Only three poroelastic parameters are involved in their formulation as against five poroelastic parameters which define a general, homogeneous, isotropic, poroelastic medium.

The aim of the present analytical study is to formulate the two-dimensional plane strain problem of the quasi-static deformation of a multilayered poroelastic half-space by surface loads. The mathematical analysis consists of two parts: (i) The derivation of the poroelastic solution of the coupled system, and (ii) the derivation of the explicit expressions for the elements of the $6 \times 6$ Haskell propagator matrix. For finding the

Keywords. Multi-layered half-space; plane strain; poroelastic; propagator matrix; surface loads. 
poroelastic solution, the stresses and the pore pressure are taken as the basic state variables. The displacements are obtained by integrating the coupled constitutive relations. The Fourier-Laplace transforms of the stresses, displacements, pore pressure and fluid flux in a homogeneous isotropic medium are expressed in terms of six arbitrary constants. The expressions for the elements of the propagator matrix obtained are simple functions of the five poroelastic parameters.

Geomechanic problems, such as loading by a reservoir lake or seabed structure that is very extensive in one direction on the Earth's surface, can be solved as two-dimensional plane strain problems. Bell and Nur (1978) used two-dimensional half-space models with surface loading to study the change in strength produced by reservoir-induced pore pressure and stresses for thrust, normal and strike-slip faulting.

\section{Basic equations}

We consider a two-dimensional approximation in which the displacement components $\left(u_{1}, u_{2}, u_{3}\right)$ are independent of the Cartesian coordinate $x_{2}$ so that $\partial / \partial x_{2} \equiv 0$. Under this assumption, the plane strain problem $\left(u_{2}=0\right)$ and the antiplane strain problem $\left(u_{1}=u_{3}=0\right)$ get decoupled and can therefore be treated independently. Since the antiplane deformation is not affected by pore pressure, we shall confine our discussion to the plane strain problem only.

A homogeneous, isotropic, poroelastic medium can be characterized by five poroelastic parameters: shear modulus $(G)$, drained Poisson's ratio $(\nu)$, undrained Poisson's ratio $\left(\nu_{u}\right)$, Skempton's coefficient $(B)$ and hydraulic diffusivity $(c)$. Darcy conductivity $(\chi)$ and Biot-Willis coefficient $(\alpha)$ can be expressed in terms of these five parameters.

For plane strain deformation of a poroelastic medium in the $x_{1} x_{3}$-plane, the displacement components in the solid skeleton are of the form

$$
\begin{aligned}
& u_{1}=u_{1}\left(x_{1}, x_{3}, t\right), \\
& u_{2}=0, \\
& u_{3}=u_{3}\left(x_{1}, x_{3}, t\right) .
\end{aligned}
$$

Let $\sigma_{i j}$ denote the total stresses in the fluid-infiltrated porous elastic material, $\varepsilon_{i j}$ the corresponding strains and $p$ the pore pressure (compression negative). For plane strain, these quantities are related through the following coupled system of equations (Rice and Cleary 1976; Roeloffs 1988) in which the total stresses $\sigma_{i j}$ and the pore pressure $p$ are taken as the basic state variables.

$$
\frac{\partial \sigma_{11}}{\partial x_{1}}+\frac{\partial \sigma_{13}}{\partial x_{3}}=0, \quad \frac{\partial \sigma_{13}}{\partial x_{1}}+\frac{\partial \sigma_{33}}{\partial x_{3}}=0 .
$$

\subsection{Compatibility equation}

$$
\nabla^{2}\left(\sigma_{11}+\sigma_{33}+2 \eta p\right)=0,
$$

where

$$
\eta=\frac{1-2 \nu}{2(1-\nu)} \alpha
$$

is the poroelastic stress coefficient.

\subsection{Pore fluid mass conservation equation}

$$
\left(c \nabla^{2}-\frac{\partial}{\partial t}\right)\left[\sigma_{11}+\sigma_{33}+\frac{3}{\left(1+\nu_{u}\right) B} p\right]=0 .
$$

\subsection{Constitutive equations}

$$
\begin{aligned}
& 2 G \varepsilon_{11}=(1-\nu) \sigma_{11}-\nu \sigma_{33}+\alpha_{0} p, \\
& 2 G \varepsilon_{33}=(1-\nu) \sigma_{33}-\nu \sigma_{11}+\alpha_{0} p, \\
& 2 G \varepsilon_{13}=\sigma_{13},
\end{aligned}
$$

where

$$
\alpha_{0}=(1-2 \nu) \alpha .
$$

Further,

$$
\begin{aligned}
\varepsilon_{21} & =\varepsilon_{22}=\varepsilon_{23}=0, \\
\sigma_{21} & =\sigma_{23}=0, \\
\sigma_{22} & =\nu\left(\sigma_{11}+\sigma_{33}\right)-\alpha_{0} p .
\end{aligned}
$$

The coupled system of equations in (2) to (5) can be solved in terms of Biot's stress function (Wang 2000). We put

$$
\begin{aligned}
\sigma_{11} & =\frac{\partial^{2} F}{\partial x_{3}^{2}}, \quad \sigma_{33}=\frac{\partial^{2} F}{\partial x_{1}^{2}}, \\
\sigma_{13} & =-\frac{\partial^{2} F}{\partial x_{1} \partial x_{3}} .
\end{aligned}
$$

The equilibrium equations in (2) are then identically satisfied. Equations (3), (5) and (12) yield

$$
\begin{gathered}
\nabla^{2}\left(\nabla^{2} F+2 \eta p\right)=0 \\
\left(c \nabla^{2}-\frac{\partial}{\partial t}\right)\left[\nabla^{2} F+\frac{3}{\left(1+\nu_{u}\right) B} p\right]=0 .
\end{gathered}
$$


Eliminating $F$ and $p$ in turn, equations (13) and (14) lead us to the following decoupled equations

$$
\begin{aligned}
& \left(c \nabla^{2}-\frac{\partial}{\partial t}\right) \nabla^{2} p=0, \\
& \left(c \nabla^{2}-\frac{\partial}{\partial t}\right) \nabla^{4} F=0 .
\end{aligned}
$$

The general solution of equation (15) may be expressed in the form

$$
p=p_{1}+p_{2},
$$

where

$$
\begin{aligned}
c \nabla^{2} p_{1} & =\frac{\partial p_{1}}{\partial t}, \\
\nabla^{2} p_{2} & =0 .
\end{aligned}
$$

Similarly, the general solution of equation (16) may be expressed in the form

$$
F=F_{1}+F_{2},
$$

where

$$
\begin{aligned}
c \nabla^{2} F_{1} & =\frac{\partial F_{1}}{\partial t} \\
\nabla^{4} F_{2} & =0 .
\end{aligned}
$$

Taking the Laplace transform of equations (18), (19), (21) and (22), we have

$$
\begin{aligned}
\nabla^{2} \bar{p}_{1}-\frac{s}{c} \bar{p}_{1} & =0, \\
\nabla^{2} \bar{p}_{2} & =0, \\
\nabla^{2} \bar{F}_{1}-\frac{s}{c} \bar{F}_{1} & =0, \\
\nabla^{4} \bar{F}_{2} & =0,
\end{aligned}
$$

where, for example,

$$
\bar{p}_{1}\left(x_{1}, x_{2}, s\right)=\int_{0}^{\infty} p_{1}\left(x_{1}, x_{3}, t\right) e^{-s t} d t
$$

is the Laplace transform of $p_{1}\left(x_{1}, x_{3}, t\right)$. In the following, we shall omit the overbar from the Laplace transform of a function. Thus, for example, $\bar{p}_{1}\left(x_{1}, x_{3}, s\right)$ can be written as $p_{1}\left(x_{1}\right.$, $\left.x_{3}, s\right)$.
For plane parallel boundaries of the form $x_{3}=$ const., suitable solutions of equations (23) to (26) are

$$
\begin{aligned}
p_{1}= & \int_{0}^{\infty}\left(A_{1} e^{-m z}+C_{1} e^{m z}\right)\left(\begin{array}{c}
\sin k x \\
\cos k x
\end{array}\right) d k, \\
p_{2}= & \int_{0}^{\infty}\left(A_{2} e^{-k z}+C_{2} e^{k z}\right)\left(\begin{array}{c}
\sin k x \\
\cos k x
\end{array}\right) d k, \\
F_{1}= & \int_{0}^{\infty}\left(B_{1} e^{-m z}+D_{1} e^{m z}\right)\left(\begin{array}{c}
\sin k x \\
\cos k x
\end{array}\right) d k, \\
F_{2}= & \int_{0}^{\infty}\left[\left(B_{2}+B_{3} k z\right) e^{-k z}+\left(D_{2}+D_{3} k z\right) e^{k z}\right] \\
& \times\left(\begin{array}{c}
\sin k x \\
\cos k x
\end{array}\right) d k,
\end{aligned}
$$

where $A_{1}, C_{1}$, etc. may be functions of $k, x=x_{1}$, $z=x_{3}$ and

$$
m=\left(k^{2}+s / c\right)^{1 / 2} .
$$

Using (17), (20), (24) to (31), equation (13) yields

$$
A_{1}=-\left(\frac{s}{2 c \eta}\right) B_{1}, \quad C_{1}=-\left(\frac{s}{2 c \eta}\right) D_{1} .
$$

Similarly, equation (14) implies

$$
\begin{aligned}
A_{2} & =\frac{2}{3}\left(1+\nu_{u}\right) B k^{2} B_{3}, \\
C_{2} & =-\frac{2}{3}\left(1+\nu_{u}\right) B k^{2} D_{3} .
\end{aligned}
$$

From equations (17), (28) and (29), we have

$$
\begin{aligned}
p= & \int_{0}^{\infty}\left(A_{1} e^{-m z}+C_{1} e^{m z}+A_{2} e^{-k z}+C_{2} e^{k z}\right) \\
& \times\left(\begin{array}{c}
\sin k x \\
\cos k x
\end{array}\right) d k .
\end{aligned}
$$

The fluid flux in the $z$-direction, $q$, is given by

$$
\begin{aligned}
q= & -\frac{\chi \partial p}{\partial z} \\
= & \chi \int_{0}^{\infty}\left[m\left(A_{1} e^{-m z}-C_{1} e^{m z}\right)\right. \\
& \left.+k\left(A_{2} e^{-k z}-C_{2} e^{k z}\right)\right]\left(\begin{array}{c}
\sin k x \\
\cos k x
\end{array}\right) d k .
\end{aligned}
$$


Using (33) and (34), equations (35) and (36) become

$$
\begin{aligned}
p= & -\int_{0}^{\infty}\left[\frac{s}{2 c \eta}\left(B_{1} e^{-m z}+D_{1} e^{m z}\right)\right. \\
& \left.+\xi k^{2}\left(-B_{3} e^{-k z}+D_{3} e^{k z}\right)\right]\left(\begin{array}{c}
\sin k x \\
\cos k x
\end{array}\right) d k, \\
q= & \chi \int_{0}^{\infty}\left[\frac{m s}{2 c \eta}\left(-B_{1} e^{-m z}+D_{1} e^{m z}\right)\right. \\
& \left.+\xi k^{3}\left(B_{3} e^{-k z}+D_{3} e^{k z}\right)\right]\left(\begin{array}{c}
\sin k x \\
\cos k x
\end{array}\right) d k
\end{aligned}
$$

where

$$
\xi=\frac{2}{3}\left(1+\nu_{u}\right) B
$$

From equations (12), (20), (30) and (31), we find

$$
\begin{aligned}
\sigma_{11}= & \int_{0}^{\infty}\left[m^{2}\left(B_{1} e^{-m z}+D_{1} e^{m z}\right)\right. \\
& +k^{2}\left(B_{2} e^{-k z}+D_{2} e^{k z}\right)+k^{2}\left\{(k z-2) B_{3} e^{-k z}\right. \\
& \left.\left.+(k z+2) D_{3} e^{k z}\right\}\right]\left(\begin{array}{c}
\sin k x \\
\cos k x
\end{array}\right) d k
\end{aligned}
$$

$$
\begin{aligned}
\sigma_{33}= & -\int_{0}^{\infty}\left[B_{1} e^{-m z}+D_{1} e^{m z}+\left(B_{2}+B_{3} k z\right) e^{-k z}\right. \\
& \left.+\left(D_{2}+D_{3} k z\right) e^{k z}\right]\left(\begin{array}{c}
\sin k x \\
\cos k x
\end{array}\right) k^{2} d k,
\end{aligned}
$$$$
\sigma_{13}=\int_{0}^{\infty}\left[m\left(B_{1} e^{-m z}-D_{1} e^{m z}\right)\right.
$$$$
+k\left(B_{2} e^{-k z}-D_{2} e^{k z}\right)+k\left\{B_{3}(k z-1) e^{-k z}\right.
$$$$
\left.\left.-D_{3}(k z+1) e^{k z}\right\}\right]\left(\begin{array}{c}
\cos k x \\
-\sin k x
\end{array}\right) k d k .
$$

Corresponding to the stresses given by equations (40) to (42), the displacements are found by integrating the coupled constitutive relations given in equations (6) to (8). We find (Singh and Garg 1985)

$$
\begin{aligned}
2 G u_{1}= & -\int_{0}^{\infty}\left[B_{1} e^{-m z}+D_{1} e^{m z}+B_{2} e^{-k z}+D_{2} e^{k z}\right. \\
& +B_{3}\left(2 \nu_{u}-2+k z\right) e^{-k z} \\
& \left.+D_{3}\left(-2 \nu_{u}+2+k z\right) e^{k z}\right]\left(\begin{array}{c}
\cos k x \\
-\sin k x
\end{array}\right) k d k, \\
2 G u_{3}= & \int_{0}^{\infty}\left[m\left(B_{1} e^{-m z}-D_{1} e^{m z}\right)\right. \\
& +k\left(B_{2} e^{-k z}-D_{2} e^{k z}\right) \\
& +B_{3}\left(1-2 \nu_{u}+k z\right) k e^{-k z} \\
& \left.+D_{3}\left(1-2 \nu_{u}-k z\right) k e^{k z}\right]\left(\begin{array}{c}
\sin k x \\
\cos k x
\end{array}\right) d k .
\end{aligned}
$$

Equations (37), (38) and (41) to (43) may be written in the form

$$
\begin{aligned}
p & =\int_{0}^{\infty} P\left(\begin{array}{c}
\sin k x \\
\cos k x
\end{array}\right) d k, \\
q & =\int_{0}^{\infty} T\left(\begin{array}{c}
\sin k x \\
\cos k x
\end{array}\right) d k, \\
\sigma_{33} & =\int_{0}^{\infty} N\left(\begin{array}{c}
\sin k x \\
\cos k x
\end{array}\right) k d k, \\
\sigma_{13} & =\int_{0}^{\infty} S\left(\begin{array}{c}
\cos k x \\
-\sin k x
\end{array}\right) k d k, \\
u_{1} & =\int_{0}^{\infty} U\left(\begin{array}{c}
\cos k x \\
-\sin k x
\end{array}\right) d k, \\
u_{3} & =\int_{0}^{\infty} W\left(\begin{array}{c}
\sin k x \\
\cos k x
\end{array}\right) d k .
\end{aligned}
$$

The functions $P(k, z, s)$, etc. are given by the matrix relation

$$
\mathbf{A}(z)=\mathbf{Z}(z) \mathbf{E}(z) \mathbf{K},
$$

where $\mathbf{A}, \mathbf{K}$ are the column vectors

$$
\begin{aligned}
\mathbf{A}(z) & =[U, W, S, N, P, T]^{T}, \\
\mathbf{K} & =\left[B_{1}, D_{1}, B_{2}, D_{2}, B_{3}, D_{3}\right]^{T},
\end{aligned}
$$

and $\mathbf{E}$ is the diagonal matrix

$$
\mathbf{E}(z)=\operatorname{diag}\left(e^{-m z}, e^{m z}, e^{-k z}, e^{k z}, e^{-k z}, e^{k z}\right) .
$$


The elements of the $6 \times 6$ matrix $\mathbf{Z}(z)$ are

$$
\begin{aligned}
& (11)=(12)=(13)=(14)=-\frac{k}{2 G}, \\
& (15)=-\left(\frac{k}{2 G}\right)\left(2 \nu_{u}-2+k z\right), \\
& (16)=-\left(\frac{k}{2 G}\right)\left(-2 \nu_{u}+2+k z\right), \\
& (21)=-(22)=\frac{m}{2 G}, \\
& (23)=-(24)=\frac{k}{2 G} \text {, } \\
& (25)=\left(\frac{k}{2 G}\right)\left(1-2 \nu_{u}+k z\right), \\
& (26)=\left(\frac{k}{2 G}\right)\left(1-2 \nu_{u}-k z\right), \\
& (31)=-(32)=m, \\
& (33)=-(34)=k, \\
& (35)=k(k z-1),(36)=-k(k z+1), \\
& (41)=(42)=(43)=(44)=-k, \\
& (45)=(46)=-k^{2} z, \\
& (51)=(52)=-s / 2 c \eta, \\
& (53)=(54)=0 \text {, } \\
& (55)=-(56)=\xi k^{2}, \\
& (61)=-(62)=-\frac{m s \chi}{2 c \eta}, \\
& (63)=(64)=0 \text {, } \\
& (65)=(66)=\xi \chi k^{3} \text {. }
\end{aligned}
$$

The $4 \times 4$ matrix obtained on deleting the first and the second columns and the fifth and the sixth rows of the matrix $\mathbf{Z}$ for a poroelastic medium given by equation (50) coincides with the corresponding matrix for an elastic medium given by Singh and Garg (1985) with the drained Poisson's ratio $\nu$ replaced by the undrained Poisson's ratio $\nu_{u}$.

\section{Multi-layered half-space}

We consider a semi-infinite poroelastic medium consisting of $j-1$ parallel, homogeneous, isotropic layers lying over a homogeneous, isotropic halfspace. The layers are numbered serially, the topmost layer being layer 1 and the half-space, layer $j$. The origin of the Cartesian system $(x, y, z)$ is placed at the surface with the $z$-axis drawn vertically downwards into the medium. The $n$th layer is of thickness $d_{n}$, the corresponding poroelastic parameters are denoted by $G_{n}, \nu_{n}, \nu_{u n}, B_{n}, c_{n}$ and is bounded by the interfaces $z=z_{n-1}, z_{n}$. Evidently, $d_{n}=z_{n}-z_{n-1}, z_{0}=0$ and $z_{j-1}=H$, where $H$ is the depth of the last interface. From equation (47), we have

$$
\begin{aligned}
\mathbf{A}_{n}(z) & =\left[U_{n}, W_{n}, S_{n}, N_{n}, P_{n}, T_{n}\right]^{T} \\
& =\mathbf{Z}_{n}(z) \mathbf{E}_{n}(z) \mathbf{K}_{n}, \quad\left(z_{n-1} \leq z \leq z_{n}\right), \\
\mathbf{K}_{n} & =\left[B_{1 n}, D_{1 n}, B_{2 n}, D_{2 n}, B_{3 n}, D_{3 n}\right]^{T} .
\end{aligned}
$$

Equation (51) yields

$$
\mathbf{A}_{n}\left(z_{n-1}\right)=\mathbf{a}_{n} \mathbf{A}_{n}\left(z_{n}\right),
$$

where $\mathbf{a}_{n}$ is the propagator matrix

$$
\mathbf{a}_{n}\left(z_{n-1}, z_{n}\right)=\mathbf{Z}_{n}\left(z_{n-1}\right) \mathbf{E}_{n}\left(z_{n-1}\right) \mathbf{E}_{n}^{-1}\left(z_{n}\right) \mathbf{Z}_{n}^{-1}\left(z_{n}\right) .
$$

To evaluate $\mathbf{a}_{n}$, we temporarily shift the origin to the interface $z=z_{n}$. This yields

$$
\mathbf{a}_{n}\left(d_{n}\right)=\mathbf{Z}_{n}\left(-d_{n}\right) \mathbf{E}_{n}\left(-d_{n}\right) \mathbf{Z}_{n}^{-1}(0) .
$$

The continuity of $u_{1}, u_{3}, \sigma_{13}, \sigma_{33}, p$ and $q$ at the interface $z=z_{n-1}$ implies

$$
\mathbf{A}_{n-1}\left(z_{n-1}\right)=\mathbf{A}_{n}\left(z_{n-1}\right) .
$$

Equation (52) can now be written in the form

$$
\mathbf{A}_{n-1}\left(z_{n-1}\right)=\mathbf{a}_{n} \mathbf{A}_{n}\left(z_{n}\right) .
$$

The propagator matrix $\mathbf{a}_{n}$ is a function of the five poroelastic parameters of the $n$th layer and its thickness $d_{n}=z_{n}-z_{n-1}$. It does not depend upon $z_{n-1}$ or $z_{n}$ per se. The elements of the matrix $\mathbf{Z}^{-1}(0)$ are given in Appendix $\mathrm{A}$ and the elements of the propagator matrix $\mathbf{a}_{n}\left(d_{n}\right)$ are given in Appendix B.

A repeated use of the relation (54) yields

$$
\mathbf{A}_{1}(0)=\mathbf{V A}_{j}(H),
$$

where

$$
\mathbf{V}=\mathbf{a}_{1} \mathbf{a}_{2} \cdots \mathbf{a}_{j-1}
$$


In the half-space (layer $j$ ), we apply the finiteness condition that the displacements, stresses and pore pressure remain finite as $z \rightarrow \infty$. This requires

$$
D_{1 j}=D_{2 j}=D_{3 j}=0,
$$

so that

$$
\mathbf{K}_{j}=\left[B_{1 j}, 0, B_{2 j}, 0, B_{3 j}, 0\right]^{T} .
$$

From equations (51), (55) and (58), we have

$$
\begin{aligned}
& {\left[U_{0}, W_{0}, S_{0}, N_{0}, P_{0}, T_{0}\right]^{T}} \\
& \quad=\mathbf{J}\left[B_{1 j}, 0, B_{2 j}, 0, B_{3 j}, 0\right]^{T},
\end{aligned}
$$

where

$$
\mathbf{J}=\mathbf{V Z}_{j}(H) \mathbf{E}_{j}(H)
$$

\section{Surface loads}

For prescribed surface loads, $S_{0}, N_{0}$ are known. Moreover, $p=0\left(P_{0}=0\right)$ at the surface $z=0$. Equation (59) gives

$$
\begin{gathered}
S_{0}=J_{31} B_{1 j}+J_{33} B_{2 j}+J_{35} B_{3 j}, \\
N_{0}=J_{41} B_{1 j}+J_{43} B_{2 j}+J_{45} B_{3 j}, \\
0=J_{51} B_{1 j}+J_{53} B_{2 j}+J_{55} B_{3 j} .
\end{gathered}
$$

These equations can be solved for $B_{1 j}, B_{2 j}, B_{3 j}$. We find

$$
B_{1 j}=\frac{\Delta_{1}}{\Delta}, \quad B_{2 j}=\frac{\Delta_{2}}{\Delta}, \quad B_{3 j}=\frac{\Delta_{3}}{\Delta},
$$

where $\Delta, \Delta_{1}, \Delta_{2}, \Delta_{3}$ are the determinants

$$
\begin{aligned}
\Delta & =\left|C_{1} C_{2} C_{3}\right|, & \Delta_{1} & =\left|C_{4} C_{2} C_{3}\right|, \\
\Delta_{2} & =\left|C_{1} C_{4} C_{3}\right|, & \Delta_{3} & =\left|C_{1} C_{2} C_{4}\right|,
\end{aligned}
$$

and $C_{1}, C_{2}, C_{3}, C_{4}$ are the columns of the $3 \times 4$ matrix

$$
\left[\begin{array}{cccc}
J_{31} & J_{33} & J_{35} & S_{0} \\
J_{41} & J_{43} & J_{45} & N_{0} \\
J_{51} & J_{53} & J_{55} & 0
\end{array}\right] .
$$

Using (62), equation (59) yields

$$
\begin{aligned}
U_{0} & =\frac{1}{\Delta}\left(J_{11} \Delta_{1}+J_{13} \Delta_{2}+J_{15} \Delta_{3}\right), \\
W_{0} & =\frac{1}{\Delta}\left(J_{21} \Delta_{1}+J_{23} \Delta_{2}+J_{25} \Delta_{3}\right) .
\end{aligned}
$$

Inserting these expressions for $U_{0}, W_{0}$ in equation (46), we can find the surface displacements $u_{1}(0), u_{3}(0)$.

\subsection{Normal strip loading}

Consider a strip $-L \leq x \leq L$ of infinite length in the $y$-direction on the surface of the multi-layered half-space. Let a normal load $\sigma_{0}$ per unit length acting in the positive $z$-direction be uniformly distributed over this strip. The boundary conditions at the surface $z=0$ are

$$
\begin{aligned}
\sigma_{13} & =0, \\
\sigma_{33} & =0 \text { for }|x|>L \\
& =-\frac{\sigma_{0}}{2 L} \text { for }|x|<L .
\end{aligned}
$$

We may write

$$
\begin{aligned}
& \sigma_{13}=0 \\
& \sigma_{33}=-\frac{\sigma_{0}}{\pi} \int_{0}^{\infty} \frac{\sin k L}{k L} \cos k x d k,
\end{aligned}
$$

for $z=0$. Comparing equations (45) and (67), we have

$$
S_{0}=0, \quad N_{0}=-\frac{\sigma_{0}}{\pi k}\left(\frac{\sin k L}{k L}\right),
$$

and the lower solution in equations (44) to (46) is to be chosen. From equations (46) and (65), we find

$u_{1}(0)=-\int_{0}^{\infty} \frac{1}{\Delta}\left(J_{11} \Delta_{1}+J_{13} \Delta_{2}+J_{15} \Delta_{3}\right)$

$\times \sin k x d k$,

$u_{3}(0)=\int_{0}^{\infty} \frac{1}{\Delta}\left(J_{21} \Delta_{1}+J_{23} \Delta_{2}+J_{25} \Delta_{3}\right) \cos k x d k$,

where $\Delta, \Delta_{1}, \Delta_{2}, \Delta_{3}$ are given by equations (63) and (64) with $S_{0}, N_{0}$ of equation (68).

\subsection{Normal line loading}

Taking the limit as $L \rightarrow 0$ with $\sigma_{0}$ fixed, equation (67) becomes

$$
\begin{aligned}
& \sigma_{13}=0 \\
& \sigma_{33}=-\frac{\sigma_{0}}{\pi} \int_{0}^{\infty} \cos k x d k=-\sigma_{0} \delta(x),
\end{aligned}
$$


at $z=0$. This represents a line load $\sigma_{0}$ per unit length acting at the origin to the surface $z=0$ in the positive $z$-direction. Equation (68) now becomes

$$
S_{0}=0, \quad N_{0}=-\frac{\sigma_{0}}{\pi k},
$$

and the lower solution is to be chosen.

\subsection{Shear line loading}

Suppose a shear line loading $\tau_{0}$ per unit length is applied at the origin to the surface $z=0$ in the positive $x$-direction. The boundary conditions at the surface $z=0$ yield

$$
\begin{aligned}
& \sigma_{33}=0, \\
& \sigma_{13}=-\tau_{0} \delta(x)=-\frac{\tau_{0}}{\pi} \int_{0}^{\infty} \cos k x d k .
\end{aligned}
$$

Comparing equations (45) and (73), we find

$$
N_{0}=0, \quad S_{0}=-\frac{\tau_{0}}{\pi k},
$$

and the upper solution is to be chosen. Equations (46) and (65) yield

$u_{1}(0)=\int_{0}^{\infty} \frac{1}{\Delta}\left(J_{11} \Delta_{1}+J_{13} \Delta_{2}+J_{15} \Delta_{3}\right) \cos k x d k$,

$u_{3}(0)=\int_{0}^{\infty} \frac{1}{\Delta}\left(J_{21} \Delta_{1}+J_{23} \Delta_{2}+J_{25} \Delta_{3}\right) \sin k x d k$,

where $\Delta, \Delta_{1}, \Delta_{2}, \Delta_{3}$ are given by equations (63) and (64) with $S_{0}, N_{0}$ of equation (74).

\subsection{Uniform half-space}

In the case of a uniform half-space $\mathbf{J}=\mathbf{Z}(0)$. Equations (50), (69), (70) and (72) yield the following expressions for the surface displacements due to a normal line load acting on the surface of a homogeneous poroelastic half-space

$$
\begin{aligned}
& u_{1}(0)=\frac{\sigma_{0}}{2 \pi G} \int_{0}^{\infty} \frac{1}{\Omega} \\
& \times\left[k-m+\left(1-2 \nu_{u}\right) \frac{s}{k \gamma_{1}}\right] \sin k x d k, \\
& u_{3}(0)=-\frac{\left(1-\nu_{u}\right) \sigma_{0} s}{\pi G \gamma_{1}} \int_{0}^{\infty} \frac{1}{k \Omega} \cos k x d k,
\end{aligned}
$$

where

$$
\begin{aligned}
& \Omega=k^{2}-m k-\frac{s}{\gamma_{1}}, \\
& \gamma_{1}=-2 c \eta \xi=2 c \frac{\left(\nu-\nu_{u}\right)}{(1-\nu)} .
\end{aligned}
$$

Similarly, for a shear line load

$$
\begin{aligned}
u_{1}(0)= & -\frac{\left(1-\nu_{u}\right) \tau_{0} s}{\pi G \gamma_{1}} \int_{0}^{\infty} \frac{1}{k \Omega} \cos k x d k, \\
u_{3}(0)= & -\frac{\tau_{0}}{2 \pi G} \int_{0}^{\infty} \frac{1}{\Omega}\left[k-m+\left(1-2 \nu_{u}\right) \frac{s}{k \gamma_{1}}\right] \\
& \times \sin k x d k .
\end{aligned}
$$

The solutions for a line load can be used as Green's functions for finding the response for general surface loading.

\section{Conclusions}

We have formulated the plane strain problem of the quasi-static deformation of a poroelastic half-space by surface loads. Poroelastic solution of the coupled system has been obtained using the Biot stress function. Explicit expressions for the LaplaceFourier transforms of the displacements, stresses, pore pressure and fluid flux in a homogeneous isotropic medium have been expressed in terms of six arbitrary constants. These expressions can be used for studying the deformation of a uniform half-space or two half-spaces in contact by surface loads or buried sources. Simplified explicit expressions for the elements of the propagator matrix have been obtained for the poroelastic case. This is a generalization of the propagator matrix obtained by Singh (1970) and Singh and Garg (1985) for the elastic case. The propagator matrix can be used for studying the quasi-static plane strain deformation of a multi-layered poroelastic half-space by surface loads or buried sources. We have found the solution in the Fourier-Laplace domain. Two integrations are required to be performed to get the solution in the space-time domain. These integrations have to be performed numerically.

\section{List of notations}

$B$ Skempton's coefficient

c hydraulic diffusivity

$d$ layer thickness

$F$ Biot's stress function

$G$ shear modulus 
$=\left(k^{2}+s / c\right)^{1 / 2}$

pore pressure (compression negative)

fluid flux in the $z$-direction

Laplace transform variable

time

$u_{i} \quad$ displacement components

$\alpha \quad$ Biot-Willis coefficient

$=\frac{3\left(\nu_{u}-\nu\right)}{B(1-2 \nu)\left(1+\nu_{u}\right)}$

$\alpha_{0} \quad=\frac{3\left(\nu_{u}-\nu\right)}{B\left(1+\nu_{u}\right)}=(1-2 \nu) \alpha$

$\beta_{1} \quad=\frac{1}{2\left(1-\nu_{u}\right)}$

$\beta_{2}=\frac{c\left(\nu-\nu_{u}\right)}{2(1-\nu)\left(1-\nu_{u}\right)}=\frac{1}{2} \gamma_{1} \beta_{1}$

$\beta_{3}=\frac{1-2 \nu_{u}}{4\left(1-\nu_{u}\right)}=\frac{1}{2}\left(1-2 \nu_{u}\right) \beta_{1}=\frac{1}{2}\left(1-\beta_{1}\right)$

$\beta_{4} \quad=\frac{B G\left(1+\nu_{u}\right)}{3\left(1-\nu_{u}\right)}=\frac{c \eta}{\chi}=G \xi \beta_{1}$

$\gamma_{1} \quad=\frac{2 c\left(\nu-\nu_{u}\right)}{(1-\nu)}=-2 c \eta \xi$

$\delta(x) \quad$ Dirac delta function

$\epsilon_{i j} \quad$ strain components

$\eta \quad=\frac{3\left(\nu_{u}-\nu\right)}{2 B(1-\nu)\left(1+\nu_{u}\right)}=\frac{1-2 \nu}{2(1-\nu)} \alpha$

$\nu \quad$ drained Poisson's ratio

$\nu_{u} \quad$ undrained Poisson's ratio

$\xi \quad=\frac{2}{3} B\left(1+\nu_{u}\right)$

$\sigma_{i j} \quad$ stress components

$\chi \quad$ Darcy conductivity

$$
=\frac{9 c\left(1-\nu_{u}\right)\left(\nu_{u}-\nu\right)}{2 G B^{2}(1-\nu)\left(1+\nu_{u}\right)^{2}}
$$

\section{Acknowledgement}

One of the authors (SJS) is thankful to the Indian National Science Academy, New Delhi for financial support under its Senior Scientist Scheme.

\section{Appendix A}

The elements of the matrix $\mathbf{Z}^{-1}(0)$ are:

$$
\begin{aligned}
& (11)=(21)=-(31)=-(41)=-2 G \beta_{2} k / s, \\
& (12)=-(22)=-2 G \beta_{2} k^{2} /(m s), \\
& (13)=-(23)=\beta_{2} k^{2} /(m s), \\
& (14)=(24)=\beta_{2} k / s \\
& (15)=(25)=-(35)=-(45)=-c \eta / s \\
& (16)=-(26)=-\beta_{4} /(m s), \\
& (32)=-(42)=G \beta_{1} / k+2 G \beta_{2} k / s, \\
& (33)=-(43)=\beta_{3} / k-\beta_{2} k / s, \\
& (34)=(44)=-1 /(2 k)-\beta_{2} k / s, \\
& (36)=-(46)=\beta_{4} /(k s), \\
& (51)=(52)=-(61)=(62)=\beta_{1} G / k, \\
& (53)=(54)=(63)=-(64)=-\beta_{1} /(2 k), \\
& (55)=(56)=(65)=(66)=0 .
\end{aligned}
$$

The constants $\beta_{i}(i=1,2,3,4)$ are defined in the List of notations.

\section{Appendix B}

The elements of the propagator matrix $\mathbf{a}_{n}\left(d_{n}\right)$ are given below (suppressing the suffix $n$ of the layer thickness $d_{n}$ and the poroelastic parameters $G_{n}, \nu_{n}, \nu_{u n}, B_{n}$ and $c_{n}$ ):

$$
\begin{aligned}
(11)= & (33)=C K D+\beta_{1} k d S K D \\
& +\frac{2 \beta_{2} k^{2}}{s}(C M D-C K D), \\
(12)= & -(43)=2 \beta_{3} S K D+\beta_{1} k d C K D \\
& +\frac{2 \beta_{2} k^{2}}{s}\left(\frac{k}{m} S M D-S K D\right), \\
(13)= & \frac{1}{2 G}\left[\left(\beta_{1}-2\right) S K D-\beta_{1} k d C K D\right. \\
& +\frac{2 \beta_{2} k^{2}}{s}\left(-\frac{k}{m} S M D+S K D\right),
\end{aligned}
$$




$$
\begin{aligned}
(14)= & -(23)=-\frac{1}{2 G} & (51)=-2 G(54)=2 G \xi \beta_{1} k(-C M D+C K D) \\
& \times\left[\beta_{1} k d S K D+\frac{2 \beta_{2} k^{2}}{s}(C M D-C K D)\right], & (52)=-2 G(53)=2 G \xi \beta_{1} k\left(-\frac{k}{m} S M D+S K D\right) \\
(15)= & \frac{1}{2 G}(45)=\frac{c \eta k}{G s}(C M D-C K D), & (55)=(66)=C M D, \\
(16)= & \frac{1}{2 G}(46)=\frac{\beta_{4}}{G s}\left(\frac{k}{m} S M D-S K D\right), & (56)=\frac{1}{m \chi} S M D \\
(21)= & -(34)=2 \beta_{3} S K D-\beta_{1} k d C K D+\frac{2 \beta_{2} k}{s} & (61)=-2 G(64)=2 c \eta k^{2}\left(S K D-\frac{m}{k} S M D\right) \\
& \times(-m S M D+k S K D), & (62)=-2 G(63)=2 c \eta k^{2}(C K D-C M D),
\end{aligned}
$$$$
(22)=(44)=C K D-\beta_{1} k d S K D+\frac{2 \beta_{2} k^{2}}{s}
$$$$
\times(-C M D+C K D)
$$$$
(24)=\frac{1}{2 G}\left[\left(\beta_{1}-2\right) S K D+\beta_{1} k d C K D\right.
$$$$
\left.+\frac{2 \beta_{2} k}{s}(m S M D-k S K D)\right],
$$$$
(25)=\frac{1}{2 G}(35)=\frac{c \eta}{G s}(-m S M D+k S K D),
$$$$
(26)=\frac{1}{2 G}(36)=\frac{\beta_{4}}{G s}(-C M D+C K D),
$$$$
(31)=-2 G \beta_{1}[S K D+k d C K D
$$$$
\left.+\frac{\gamma_{1} k}{s}(m S M D-k S K D)\right] \text {, }
$$$$
(32)=-(41)=-2 G \beta_{1}[k d S K D
$$$$
\left.+\frac{\gamma_{1} k^{2}}{s}(C M D-C K D)\right]
$$$$
(42)=2 G \beta_{1}[k d C K D-S K D
$$$$
\left.+\frac{\gamma_{1} k^{2}}{s}\left(\frac{k}{m} S M D-S K D\right)\right],
$$

where

$$
\begin{array}{ll}
C K D=\cosh (k d), & S K D=\sinh (k d), \\
C M D=\cosh (m d), & S M D=\sinh (m d) .
\end{array}
$$

The $4 \times 4$ matrix obtained by deleting the fifth and the sixth columns and the fifth and the sixth rows of $\mathbf{a}_{n}$ and putting $\nu_{u}=\nu$ in the surviving $4 \times 4$ matrix coincides with the propagator matrix for an elastic body given by Singh and Garg (1985). We have obtained the elements of the propagator matrix directly by using the definition given in equation (53). Pan (1999) obtained these elements by using the Laplace transform technique. On comparison, we find that the elements (14), (16), (23), (25), (35), (46), (52), (53), (61), (64) given by Pan (1999, Appendix III) are incorrect. To get the correct elements, the algebraic expressions appearing in lines 8 and 11, p. 1649 (Appendix III) of Pan (1999) should be replaced by the expressions

$$
\frac{1}{2 G}\left[\rho_{1} \operatorname{ch}(\lambda z)-\rho_{1} \operatorname{ch}(\lambda \rho z)-\rho_{2} \lambda z \operatorname{sh}(\lambda z)\right]
$$

and

$$
\frac{\rho_{4} \alpha_{2}}{\kappa}[\operatorname{ch}(\lambda \rho z)-\operatorname{ch}(\lambda z)]
$$

respectively.

\section{References}

Bell M L and Nur A 1978 Strength changes due to reservoirinduced pore pressure and stresses and application to Lake Oroville; J. Geophys. Res. 83 4469-4483.

Biot M A 1941 General theory of three-dimensional consolidation; J. Appl. Phys. 12 155-164.

Biot M A 1956 General solutions of the equations of elasticity and consolidation for a porous material; J. Appl. Mech. 78 91-98. 
Kalpna and Chander R 1997 On the 2D plane strain problem for a harmonic stress applied to an impervious elastic layer resting on a porous elastic half space; Phys. Earth Planet. Int. 103 151-164.

Pan E 1999 Green's functions in layered poroelastic half-spaces; Int. J. Numer. Anal. Meth. Geomech. 23 1631-1653.

Rice J R and Cleary M P 1976 Some basic stress diffusion solutions for fluid-saturated elastic porous media with compressible constituents; Rev. Geophys. Space Phys. 14 $227-241$.

Roeloffs E A 1988 Fault stability changes induced beneath a reservoir with cyclic variations in water level; J. Geophys. Res. 93 2107-2124.
Rudnicki J W 2001 Coupled deformation-diffusion effects in the mechanics of faulting and failure of geomaterials; Appl. Mech. Rev. 54 483-502.

Singh S J 1970 Static deformation of a multilayered half-space by internal sources; J. Geophys. Res. $\mathbf{7 5}$ $3257-3263$.

Singh S J and Garg N R 1985 On two-dimensional elastic dislocations in a multilayered half-space; Phys. Earth Planet. Int. 40 135-145.

Wang H F 2000 Theory of Linear Poroelasticity (Princeton: Princeton Univ. Press) p. 287.

Wang J and Fang S 2003 State space solution of nonaxisymmetric Biot consolidation problem for multilayered porous media; Int. J. Engg. Sci. 41 1799-1813.

MS received 12 August 2005; revised 14 March 2006; accepted 16 March 2006 